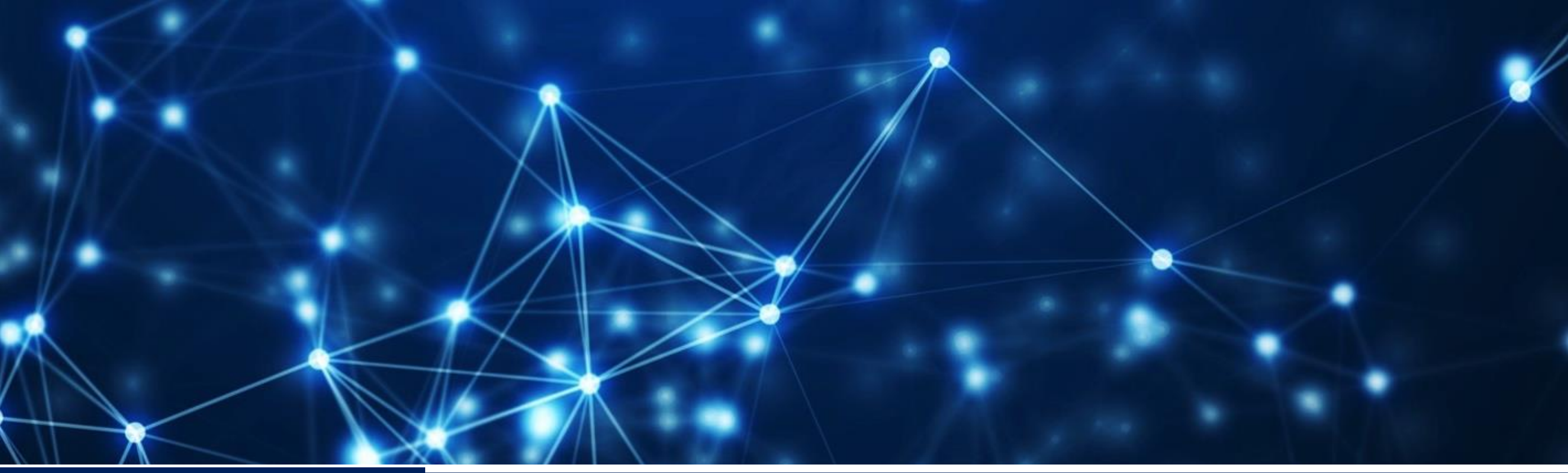

DEFENCE AND SPACE

\title{
Towards Demonstration of Photonic Payload for Telecom Satellites
}

J. Anzalchi, J. Wong, T. Verges, O. Navasquillo, T. Mengual, M. A. Piqueras, E. Prevost, K. Ravel, N. Parsons, M. Enrico, J. Bauwelink, M. Vanhoeecke, A. Vannucci, M. Tienforti International Conference on Space Optics - ICSO 2018 08 - 12 October 2018, Chania, Greece 
Outline

- Photonics Payload Concept and Benefits

- OPTIMA - Photonics Payload Demonstrator

- Photonics Technologies in vHTS Missions

- Requirements for OPTIMA Demonstrator

- Demonstrator Architecture and Demonstrated Technologies

- Photonic Frequency Generator Unit (PFGU)

- Photonic Multi Frequency Convertor (PMFC)

- Optical to Electrical Module (OEM)

- OPTIMA Current Status

- OPTIMA Possible IOD Approach

- Conclusions 


\section{Photonics Payload Concept and Benefits}

\section{- Concept:}

- Spin-in terrestrial RF-over-Fiber techniques to the core of telecoms Satellites payloads

\section{- Benefits:}

- Compact, lightweight and broadband

- Unprecedented savings on SWaP

- Ultra-wideband capacity opens path to new concept of flexibility

- No EMI/EMC issues

- Large MxN switch matrices for maximum connectivity

- Seamless integration of optical feeders

- Much simpler AIT (reduced overall cost and integration time)

- Unified operating frequency in $\mathrm{Ku}, \mathrm{Ka}$ and potentially $\mathrm{V}$ band
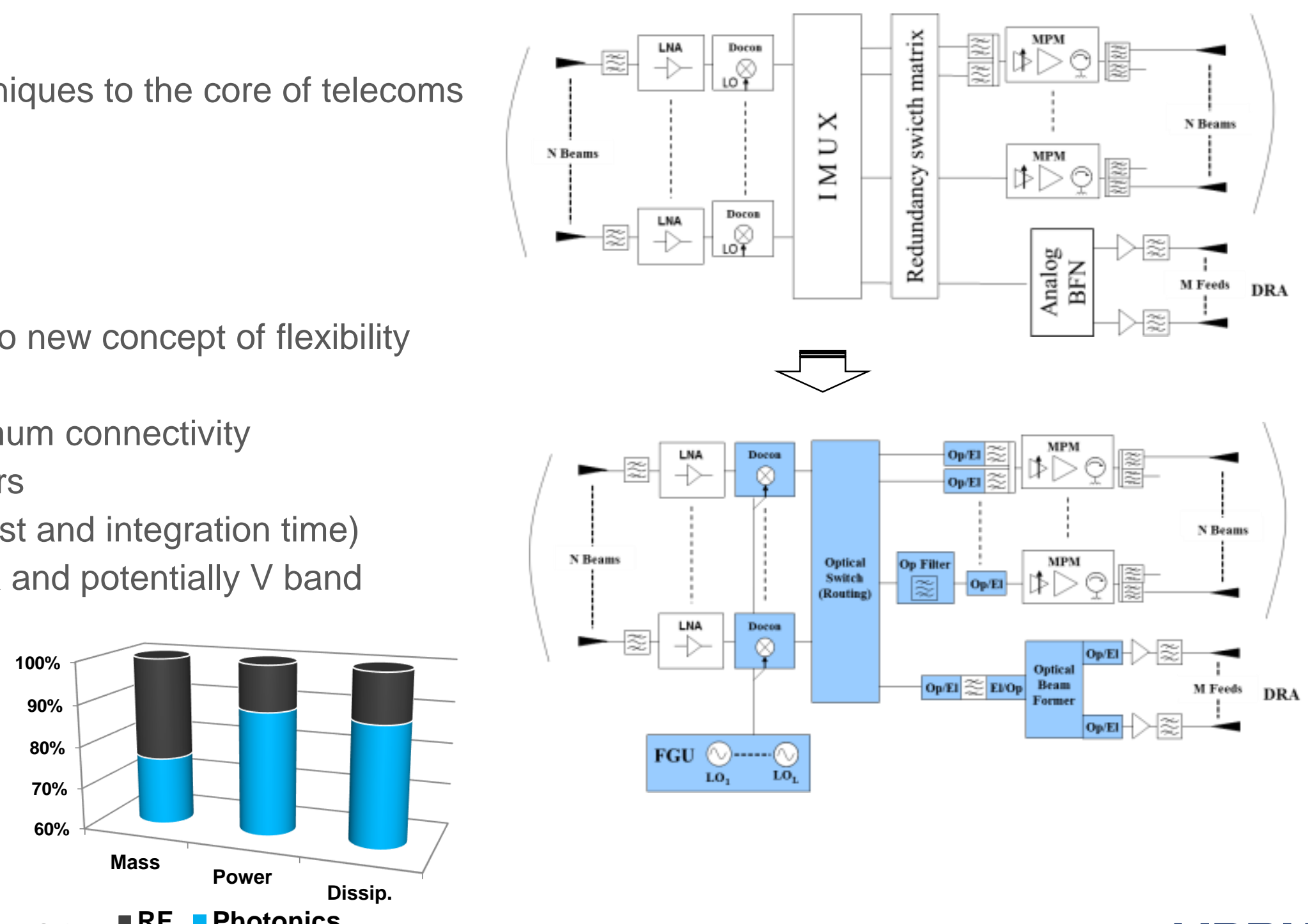
DEFENCE AND SPACE

\section{OPTIMA - Photonics Payload Demonstrator}

OPTIMA: Horizon 2020, COMPET-2-2016, EC Funded, 24-months project duration.

Kick-off: November 2016 in Brussels.

Mid-term Review: 30th November 2017 in Paris.

- Consortium:

- AIRBUS (UK lead, FR), SODERN (FR), Huber \& Suhner Polatis (UK), IMEC (BE), DAS Photonics (SP), Cordon Electronics (IT)

- Objectives:

- Demonstrate and validate to TRL6 the photonics payload concept and its benefits in a relevant industrial environment

- Build a demonstrator using building blocks from European Partners

- Test the demonstrator in ADS (Portsmouth, UK) like a real payload

- Establish specifications of each building block of future photonics payload

- Establish a roadmap towards in-orbit demonstration of photonics payload in the 2021-2023 window - Prepare future collaborations with EC and ESA up to IOD/IOV

- Increase general knowledge and raise the interest for photonics payload among the space industry - Create an industrial network and make photonics payload a reality 


\section{Photonics Technologies in vHTS Missions}

- vHTS missions form the basis for defining requirements for OPTIMA Demonstrator

- Study and RFIs have been conducted on vHTS missions from

- Terabit/s Study

- Recent RFIs from customers

- Table below shows parameters from vHTS missions that have been studied:

\begin{tabular}{|l|l|l|l|}
\hline & \multicolumn{1}{|c|}{ Terabit Study } & \multicolumn{1}{c|}{ Mission 1 } & \multicolumn{1}{c|}{ Mission 2 } \\
\hline No. of satellites & 1 & 1 & 3 \\
\hline No. of beams & 260 & 359 & 300 \\
\hline $\begin{array}{l}\text { Frequency bands of } \\
\text { operation }\end{array}$ & $\begin{array}{l}\mathrm{Q} / \mathrm{V} \text { \& Ka gateways, } \\
\text { Ka users }\end{array}$ & $\begin{array}{l}\mathrm{Q} / \mathrm{V} \text { \& Ka gateways, } \\
\text { Ka users }\end{array}$ & Q/V \& Ka gateways, Ka users \\
\hline No. of gateways & $33+4$ & $18-20$ & $16+1$ \\
\hline DC power (kW) & $\sim 25$ & $\sim 22-23$ & $\sim 21-24$ (per satellite) \\
\hline Dissipation $(\mathrm{kW})$ & $\sim 15$ & $\sim 17$ & $\sim 10-13$ (per satellite) \\
\hline Payload mass $(\mathrm{kg})$ & $\sim 2580$ & $\sim 2290-2690$ & $\sim 1860-2130$ (per satellite) \\
\hline Total capacity $(\mathrm{Gbps})$ & $\sim 1036$ & $\sim 442-504$ & $\sim 504-520$ (per satellite) \\
\hline
\end{tabular}

- The scale of vHTS missions reinforce the benefits of adopting photonic technologies

- Preliminary study has revealed a potential saving of $25 \%$ on mass and up to $15 \%$ on power consumption compared to an equivalent RF payload 


\section{Defining Requirements for OPTIMA Demonstrator}

\section{Commonalities across vHTS missions}

- The use of Ka-band and Q/V-band

- High number of beams, between 260 to 360 beams

- Frequency reuse

- High mass, equipment counts, power consumption and thermal dissipation

- Large redundancy switch matrixes

- Flexibility of switching and routing of channels

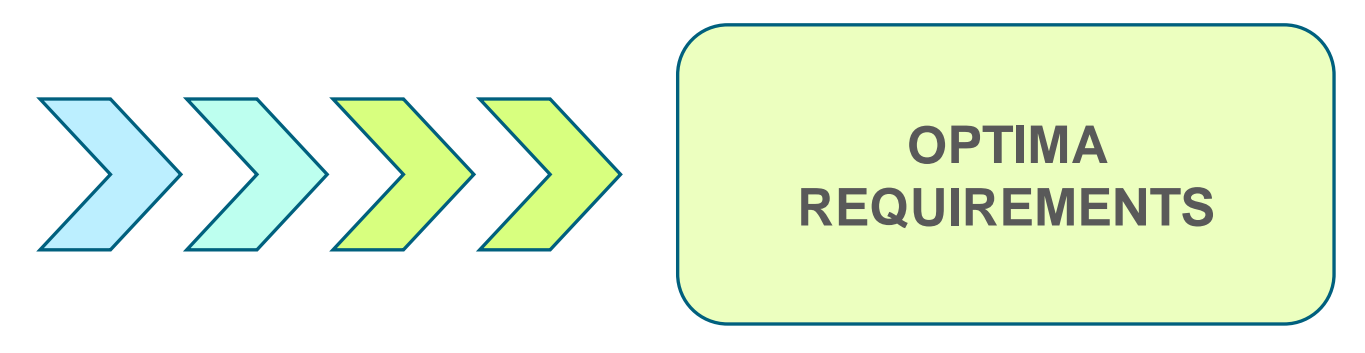




\section{Defining Requirements for OPTIMA Demonstrator}

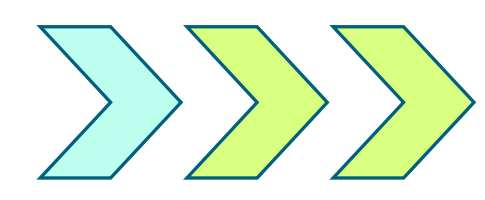

\section{OPTIMA Requirements}

- Photonics solutions to meet demanding requirements of VHTS missions.

- Demonstrator testing to verify that equivalent environmental and RF end-to-end performances can be met

- Photonic component selection and packaging techniques to reduce mass, power consumption and comply with space reliability requirements.

- Efficient frequency reuse scheme in the photonic frequency converter and multiplexing of signals for transmission in a single fibre optic cable.

- Optical switching to route any input signal to any output essential in a flexible multi beam mission. 


\section{OPTIMA Demonstrator Architecture and Demonstrated Technologies}

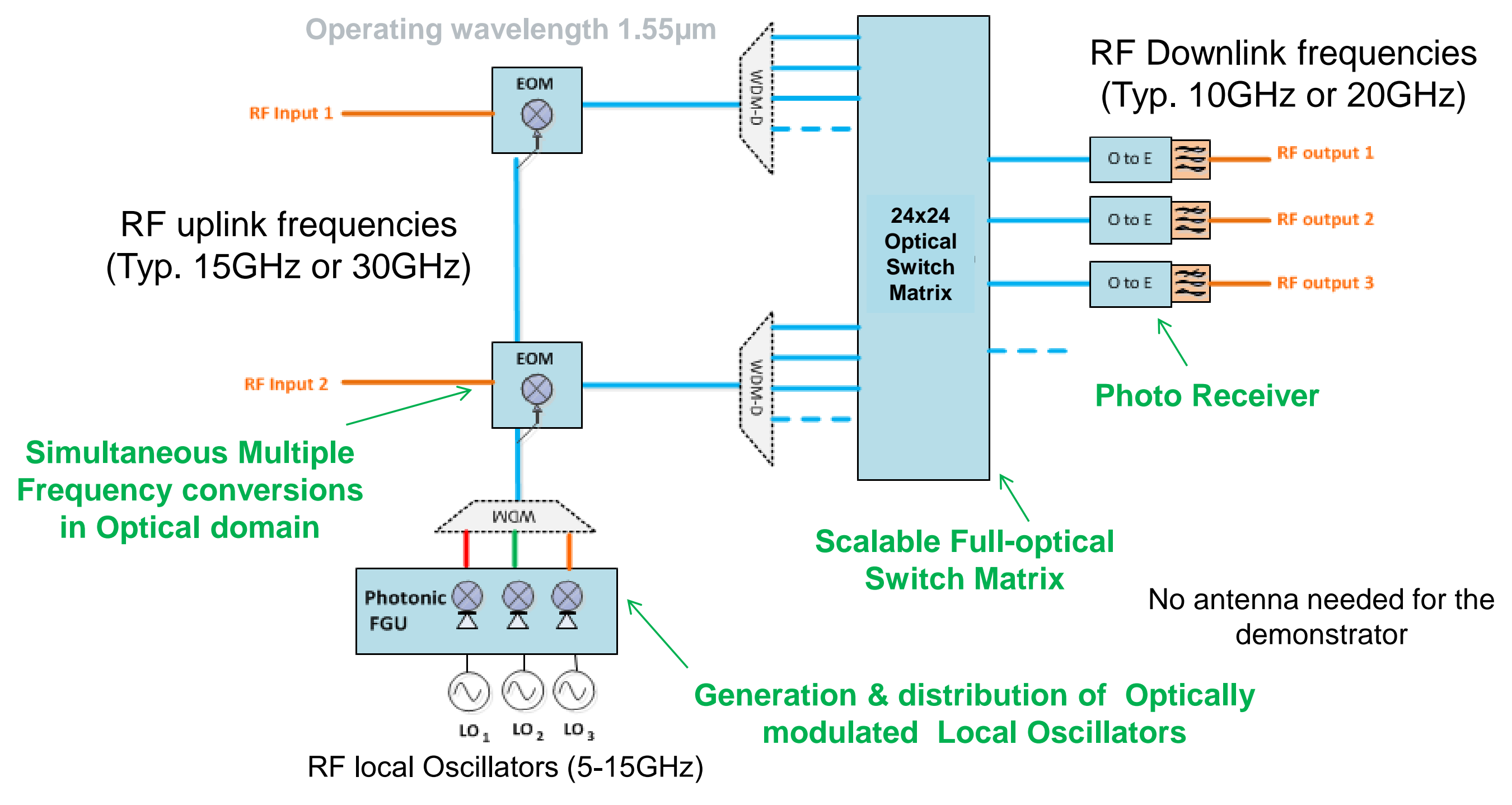




\section{Photonic Frequency Generator Unit (PFGU)}

- The PFGU is based on external modulation that converts an electrical LO to the optical domain,

- Contains an optical amplifier

- The module comprises also the control, biasing and monitoring electronics

- Particular effort has been devoted to the miniaturization of photonic modules and a co-packaged LaserModulator has been developed by Cordon Electronics

- The package is hermetically sealed. The measured RF response confirms operation up to $40 \mathrm{GHz}$

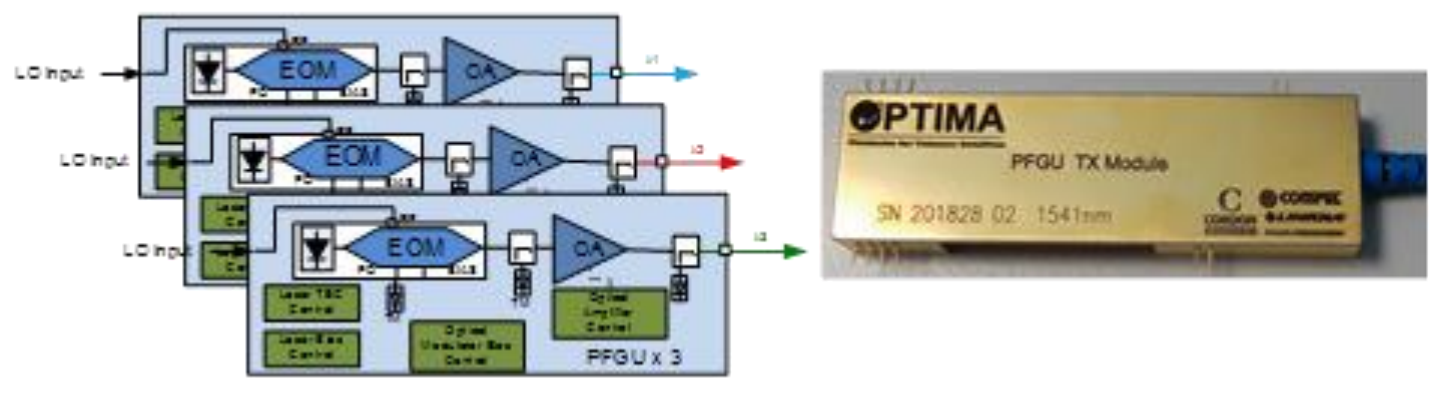

PFGU Module
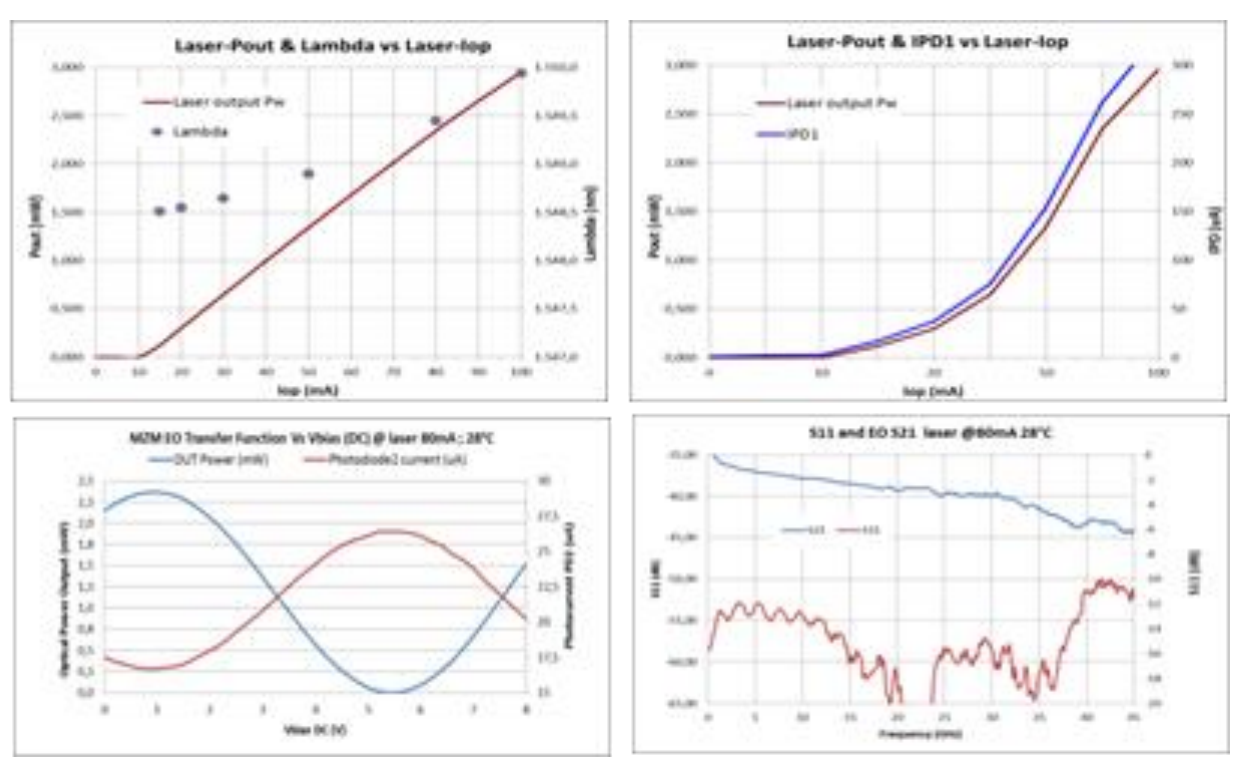


\section{Photonic Multi Frequency Convertor (PMFC)}

- The PMFC mixes the photonic LO with an RF signal.

- The mixing process is performed by an optical modulator that is in charge of modulating the RF signal on the wavelengths generated by the PFGU.

- This assembly also integrates optical amplification (EDFA) for power conditioning.

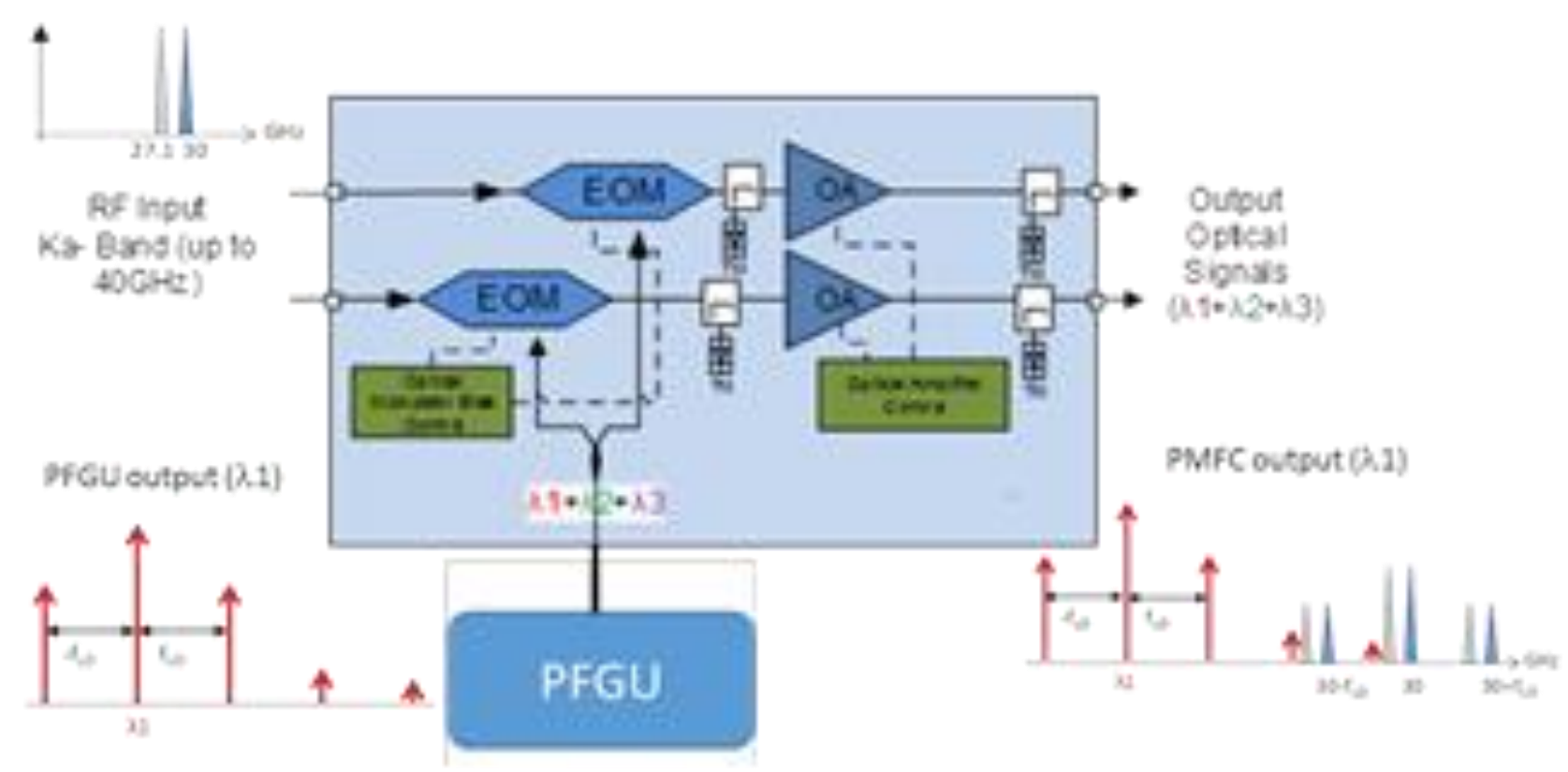

PMFC Module 
DEFENCE AND SPACE

\section{Optical to Electrical Module (OEM)}

- The OEM consists of InGaAs PIN photodetector (sensitivity for the $1250 \mathrm{~nm}$ through $1650 \mathrm{~nm}$ wavelength range) and the electronic circuitry

- The coplanar waveguide photodiode optimizes speed up to $40 \mathrm{GHz}$ ( >30GHz OPTIMA specifications)

- The desired mixing product is selected by a RF filter at the OEM output prior to be amplified and fed to the antenna.

- As this filter is in the RF domain, the photonic payload can be used at any RF, LO and IF frequency.

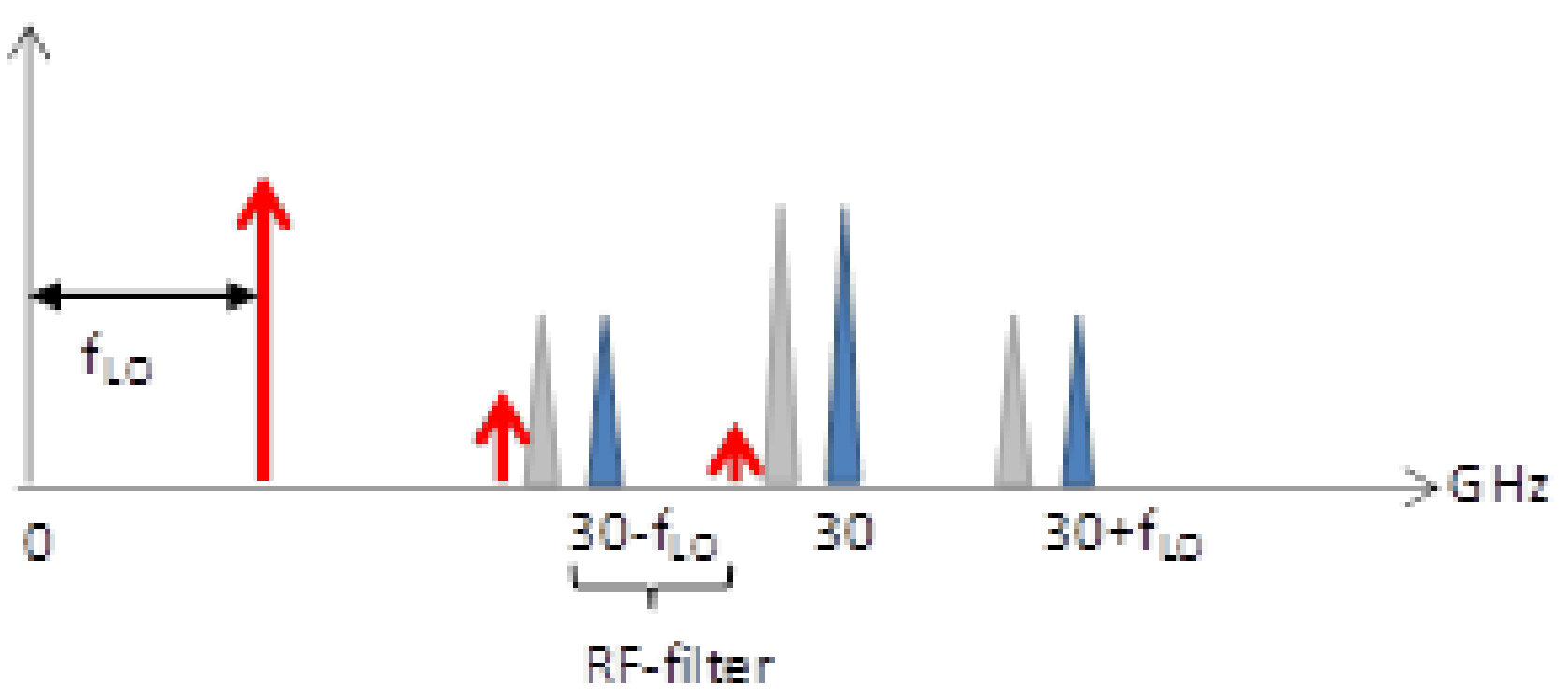

OEM Output Spectrum 


\section{Optical Switch Matrix (H+S Polatis, Sodern)}

- The optical switch matrix function is to route any input to any output essential in a flexible multi beam mission

- The current Polatis fibered optical switch matrix addresses the terrestrial telecom and datacenter markets, with a core technology currently supporting up to $384 \times 384$ ports

- Each input fiber is terminated by an optical collimator to generate a parallel beam. The collimator orientation is piloted by a 2D piezoelectric actuator. The link between two ports is established when input collimator and output collimator are aligned facing each other

- Objectives at Switch level:

- to validate that the Polatis DirectLight ${ }^{\circledR}$ technology fits for space use

- to design, manufacture, test and qualify a 48-port Switch Core Unit breadboard (24 inputs - 24 outputs) with Polatis COTS EEE (Thermal Vacuum, vibrations, shocks),

- to deliver the breadboard for implementation and testing in the OPTIMA Payload Demonstrator to demonstrate steps to TRL 6 .
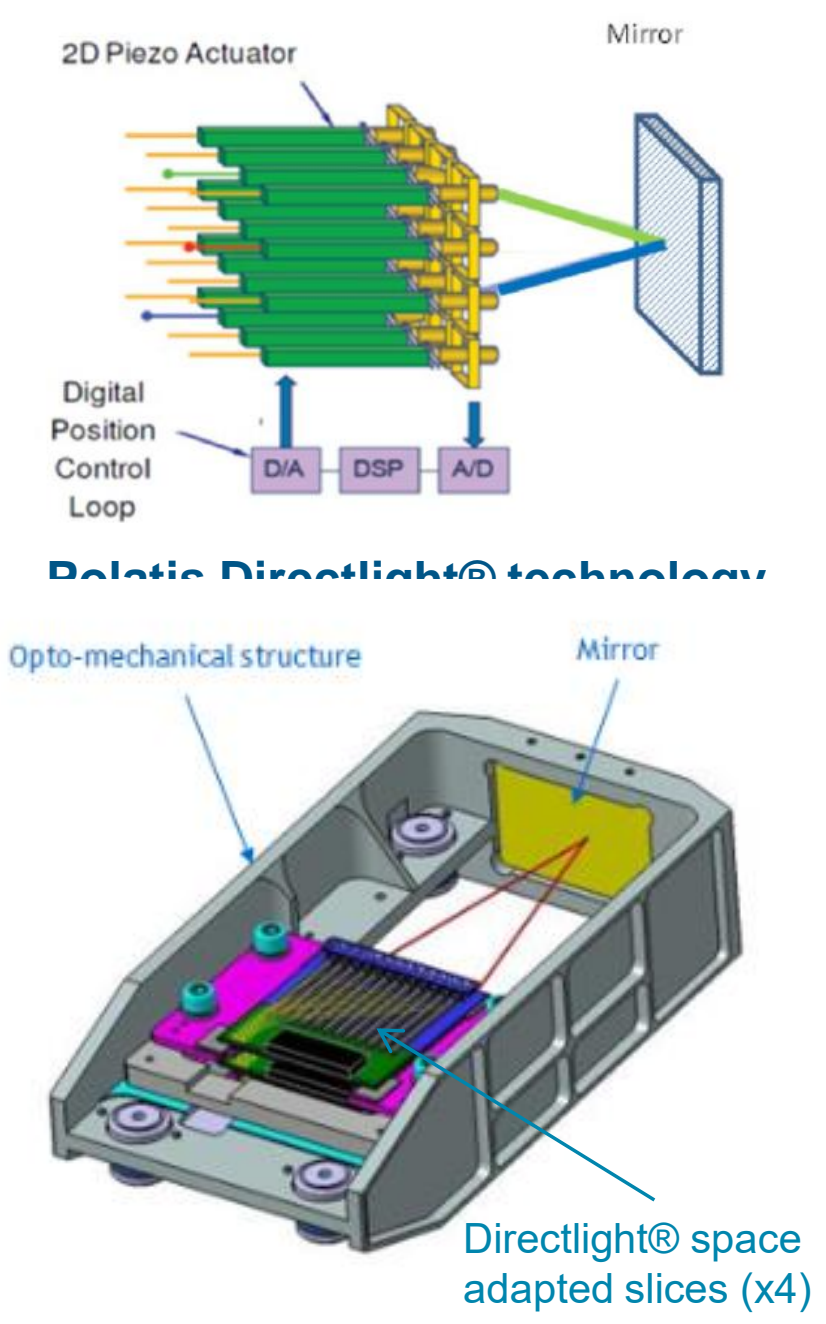

Sodern Switch Core Unit design 


\section{OPTIMA: Current Status (1/2)}

Photonic Multi-frequency Convertor, Photonic Frequency Generator Unit, Optical to RF Module Status:

- Activities Performed:

- Procurement of Photonic Parts

- Co-packaged PFGU designed, fabricated and tested

- Mechanical design and manufacturing drawings

- Electronic schematics, PCB routing and components placement

- EGSE (DC/DC board + Control board) Design and Manufacturing

- Development of the CAN BUS (according to ESA standard) driver

- BOM definition
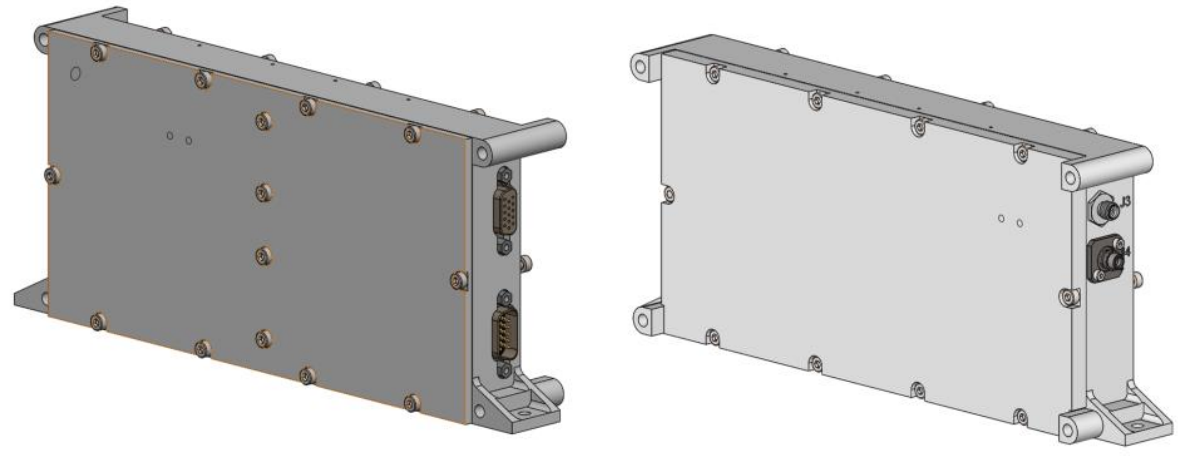

PFGU Modules

- Activities On-going

- Verification of the EGSE and CAN BUS driver

- Thermal analysis for verification purposes

- PFGU, PMFC and OEM assembly

- Next Activities:

- Modules characterisation and environmental tests

- Demonstrator set up and verification

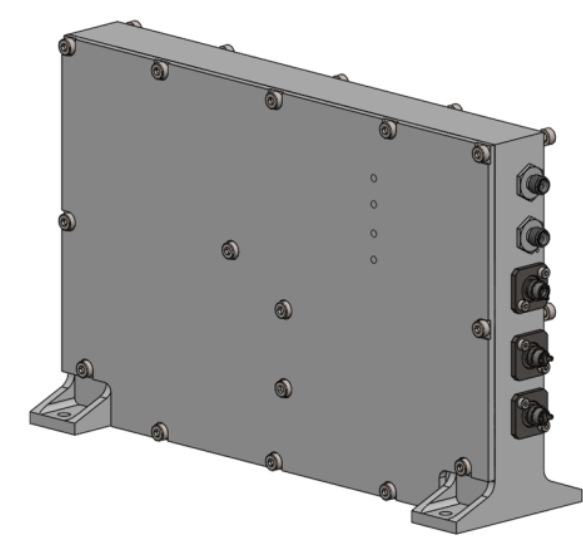

PMFC Module

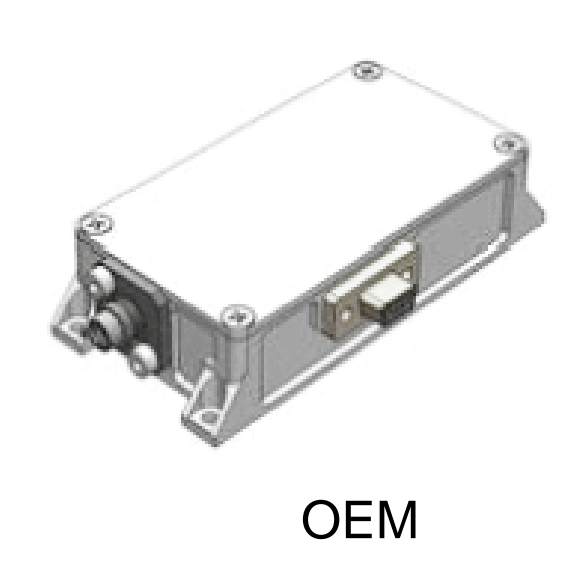




\section{OPTIMA: Current Status (2/2)}

\section{Optical Switch Matrix:}

- Activities Performed:

- Directlight ${ }^{\circledR}$ fibered core technology space design validated

- Successful vibration test (16gRMS RV) of Switch Core Unit

- Successful thermal vacuum (8 cycles $-40^{\circ} \mathrm{C} /+70^{\circ} \mathrm{C}$ non op) of Switch Core Unit

- Successful shock tests (1300g) of Switch Core Unit

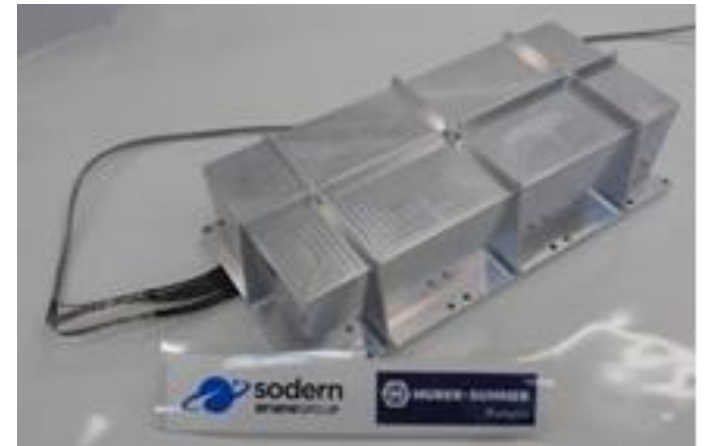

48-port switch matrix breadboard

- Terrestrial ASIC of Switch control board radiation evaluation (successful for TID 27krad, not successful for SEE 5.7 $\mathrm{MeV} . \mathrm{cm}^{2} / \mathrm{mg}$ )

- Activities On-going

- Fine electrical and optical performances final verification - high-voltage CMOS ASIC redesign to sustain radiations

- Next Activities:

- delivery to DAS Photonics for integration in OPTIMA demonstrator

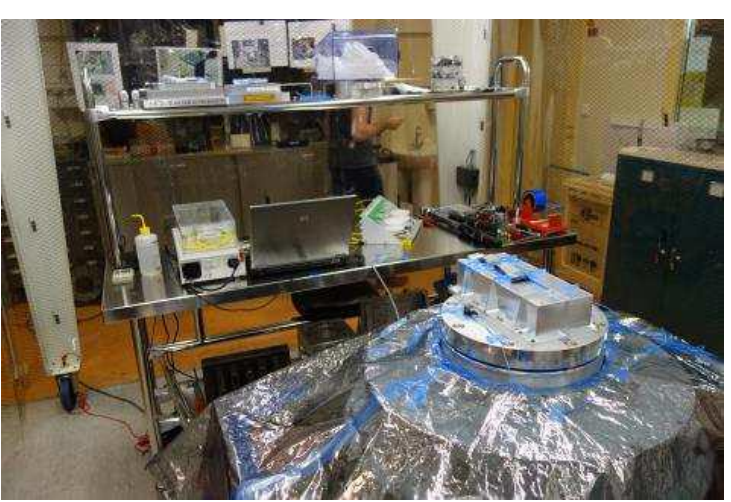

switch matrix SEB Vibration test

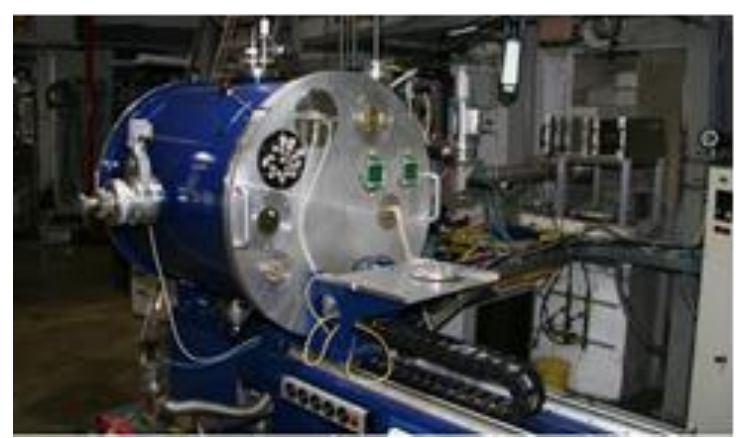

switch matrix ASIC radiation test 
DEFENCE AND SPACE

\section{OPTIMA Budgets}

- Mass for the OPTIMA payload demonstrator: $8.5 \mathrm{Kg}$

- Power consumption for the OPTIMA payload demonstrator: $54.5 \mathrm{~W}$

- TMTC:

- CAN BUS

- RS 232, RS 422 or SpW available 


\section{OPTIMA Possible IOD Approach}

- Baseline: Redundant paths to large HTS payload

- Inputs need to be connected to a switch after LNAs of an existing payload

- Outputs to be connected to a switch before HPA section

- RF LOs could be generated internally or distributed by the payload

- Alternative 1: Single purpose demonstrator

- RxTx Antenna, LNA and HPA to be added (with adequate filing and ground station)

- Alternatively can be connected to an on-board signal generator and measurement device

- Alternative 2: Multi-purpose demonstrator

- Used as payload intermediate section in complement of an RF experiment 


\section{OPTIMA IOV/IOD Schedule}

- Building blocks of OPTIMA will reach TRL6 by mid-2019

- IOV IOD as early as 2021 can be envisaged if well-defined before the end of OPTIMA

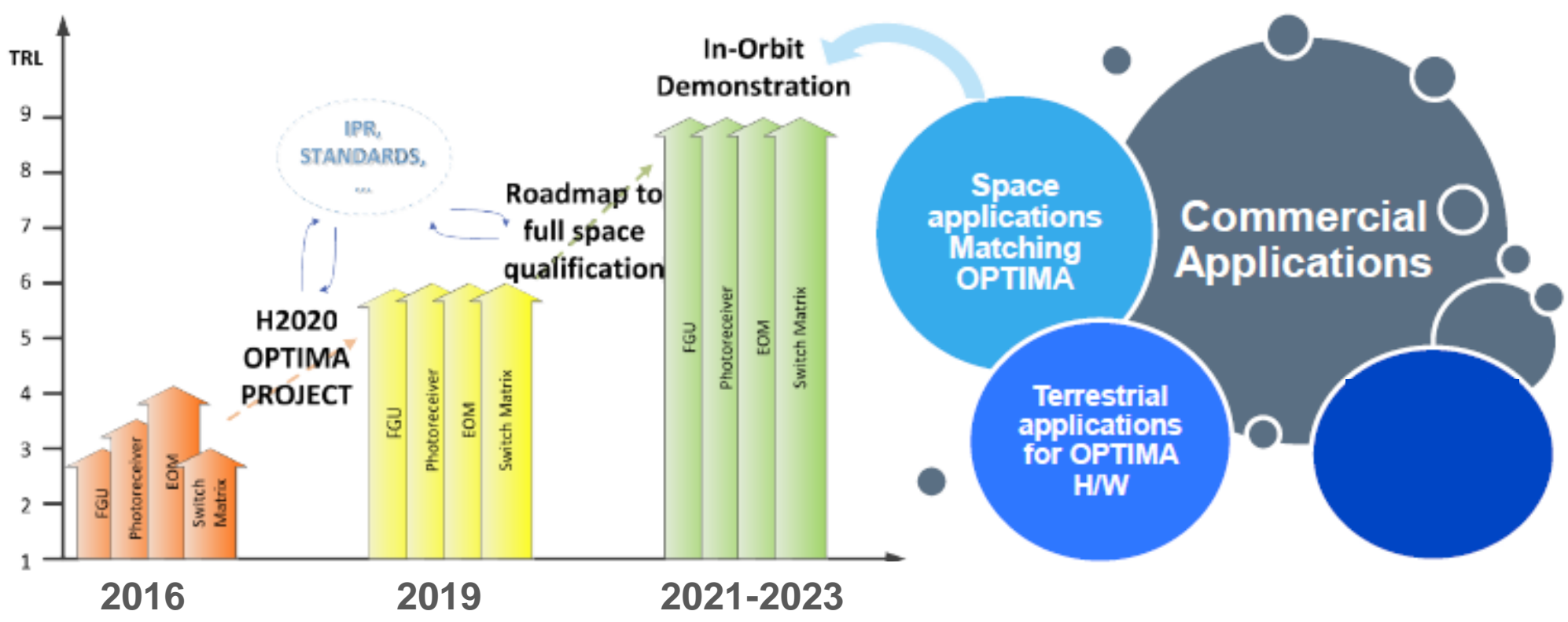

- Leveraging on the Optima project, Airbus and project partners will continue to:

- Support further TRL increase of photonics payload equipment

- In-Orbit Demonstration/Validation to Bring-Into-Use payload 


\section{Conclusions}

- Brief outline of the progress and development status of OPTIMA project has been presented.

- Recent vHTS RFIs and studies have been taken into account to collate most up-to date payload requirements specification.

- The optical switch matrix has passed the vibration, shock and thermal vacuum tests successfully

- The other equipments in the photonic payload chain are going through the final stages of assembly and testing.

- The successful environmental testing of the OPTIMA optical switch matrix breadboard paves the way to the development of a space version of the DirectLight $\AA^{\circledR}$ switching technology.

- An EGSE simulating the PDU, PSU and implementing the TT\&C system has been developed in order to fully validate functionality of the developed PFGU, PMFC, OSM and OEM modules. 
Thank you for your attention

Javad.anzalchi@airbus.com 